\title{
A Study of Combined Peak-Searching Method for Symmetric Zero Area and Gaussian Product Function Based on $\gamma$-ray Spectrum
}

\author{
He Jianfeng ${ }^{1,2,4, \text { a, * }}$, Huang Sheng ${ }^{3, \mathrm{~b}}$, Ye Zhixiang, c, Wang Xueyuan ${ }^{2, \text { a }}$, Li Xiang ${ }^{1, \mathrm{~d}}$, Zhou \\ Shirong ${ }^{1, a}$ \\ ${ }^{1}$ Fundamental Science on Radioactive Geology and Exploration Technology Laboratory, East China Institute \\ of Technology, Nanchang, Jiangxi, China \\ ${ }^{2}$ Jiangxi Engineering Laboratory on Radioactive Geoscience and Big Data Technology, East China \\ University of Technology, Nanchang, Jiangxi, China \\ ${ }^{3}$ Jiangxi Food and Drug Administration,Nanchang, Jiangxi, China \\ ${ }^{4}$ Jiangxi Engineering Technology Research Center of Nuclear Geoscience Data Science and System, East \\ China University of Technology, Nanchang, Jiangxi, China \\ ahjf_10@yeah.net, bjxhs89@163.com, 'zxye@ecit.cn ${ }^{\mathrm{d}}$ lixiang@ecit.cn
}

Keywords: $\gamma$-ray Energy Spectra, Gaussian Product Function Method, Symmetric Zero Area Method, Combined Peak-Searching

\begin{abstract}
The peak position of each peak is determined accurately in the spectra, which is a key problem in the analysis of $\gamma$-ray spectrum. In this paper, basis of comparing the Gaussian product function method and the symmetrical zero area method, the IAEA-approved G-1200 is selected as the peak-searching test spectra, the simulation experiment based on MATLAB is used for the test analysis and to extract the corresponding characteristic parameters of the spectral line. Aiming at the respective advantages of the two peak searching methods in $\gamma$-ray spectrum analysis, a method of combined peak-searching for symmetric zero area and Gaussian product function is proposed, which the statistical fluctuation of spectral data is effectively reduced, the background effect is eliminated, the peak positions information is highlighted, and the true peaks or false peaks can be identified according to the preset conditions. The result of experiment represents that symmetric zero area and Gaussian product function combined peak-searching method to peak-searching is also suitable for unsmooth data, this method has the advantages of small calculation amount, fast searching speed and obvious effect.
\end{abstract}

\section{Introduction}

The peak position of each peak in the spectra is accurately determined to be critical in gamma spectrometry. In the qualitative analysis of nuclear nucleus, it is clear that only if all the peak positions in the spectrum are correctly identified, it can be determined whether there is a certain species of nuclide in the measured sample according to the energy of the main peaks and each verification peak $^{[1-2] .}$ At the same time, the peak positions of the spectrum have a decisive influence on the determination of peak boundary, the calculation of peak area and the decomposition of overlapping peaks in the quantitative analysis of radionuclides. If the identified peaks have large errors, false peaks are mixed or real peaks are missing, which will result in the decomposition of adjacent peaks or overlapping peaks are more difficult. Therefore, in order to determine the peaks of spectrum which are very close to each other, identify weak peaks on the high background, improve the resolving ability of overlapping peaks, and eliminate false peaks, it is necessary to study the peak-searching method.

Due to the nuclear radiation measurement data approximately obeys the Gaussian distribution $f(x)=(1 / \sqrt{2 \pi \bar{n}}) \exp \left(-\frac{1}{2}(n-\bar{n})^{2} / \bar{n}^{2}\right)=(1 / \sqrt{2 \pi \bar{n}}) \exp \left(-\frac{1}{2}(x-\mu)^{2} / \sigma^{2}\right)$, two of the decisive parameters are the mathematical expectation $(\mu)$ and the variance $(\sigma)$, and $\mu$ represents the position of the Gaussian distribution that is the peak position; $\sigma$ reflects the width of the Gaussian 
distribution that is the resolution. In this paper, on the basis of comparing Gaussian product function peak-searching method and symmetric zero area transform peak-searching method, G-1200 is used as peak-searching test spectrum, MATLAB simulation experiment is used to the test analysis and to extract the corresponding characteristic parameters. Combining the advantages of the two peaksearching methods in gamma energy spectrum analysis, a symmetric zero area Gaussian function convolution method is proposed. This method can effectively reduce the statistical fluctuation of spectrum data and weaken the influence of the background. In addition, the peak position information is highlighted, and the true peaks and the false peaks can be identified based on preset conditions.

\section{The peak-searching of Gaussian product function method}

\subsection{Gaussian product function Method}

Gaussian function is usually used to describe the function of the peak shape, the expression: $g(i)=\frac{A}{\sqrt{2 \pi} \sigma} \exp \left[-\left(i-i_{0}\right)^{2} / 2 \sigma^{2}\right]$. Then a Gaussian product function $g_{h}(i)$ related to $H$ $(\mathrm{FWHM}=2.3556 \sigma)$ is defined by the adjacent data points:

$$
g_{h}(i)=\frac{g(i) g(i-1)}{g(i-2) g(i+1)}=\exp \left(\frac{11.092}{H^{2}}\right)
$$

From the above formula, the peaks of spectral are determined theoretically. When there are no peaks in the spectrum, i.e. when $H$ tends to infinity, $g_{h}(i)=1$; when there are peaks in the spectrum, i.e. when $H$ is a finite value, $g_{h}(i)=C, C$ is a constant greater than one.

Similarly, a more general Gaussian product $g_{h m}(i)$ can be defined:

$$
g_{h m}(i)=\frac{g(i) g(i+m-2)}{g(i-2) g(i+m)}=\exp \left(\frac{11.092 m}{H^{2}}\right)
$$

The parameter $m$ is the order of the Gaussian product function which is the step size (denote by channel width), then $g_{h m}(i)$ is called the maths order Gaussian product function. The sensitivity of peak-searching is related to $m$, and the sensitivity increases with the increase of $m$.

The spectrum peaks are determined theoretically from the above equation. When the spectrum has no peaks, that is, when $H$ tends to infinity, $g_{h m}(i)=1$; when the spectral has peaks, that is, $H$ is a finite value, $g_{h m}(i)=d, d$ is a constant greater than one.

In fact, due to the following two reasons: (1) the existence of the background, (2) the existence of statistical fluctuations, $g_{h m}(i)$ is not absolutely equal to 1 when the spectrum does not have a peak ${ }^{[2]}$. Therefore, in order to avoid the influence of the background parameters, it is better to use the Gaussian product function to find the peak after deducting the background. In practical applications, taking into account the influence of statistical fluctuations, the researchers turned 1 that judged the absence of spectral peaks into a "unit band."

\subsection{The peak-searching process of the Gaussian product function method}

In summary, the peak-searching process of the Gaussian product function method is specifically described as follows: firstly, according to formula (2), the Gauss product value $g_{h m}(i)$ of each point can be calculated; then the Gaussian product value is used as the basis for judging whether there are spectral peaks(when the spectrum has no peaks, that is, when $H$ approaches infinity, $g_{h m}(i)=1 \pm$ $\frac{k}{\sqrt{\text { data }_{i}}}$; when the spectral line has peaks, that is, when $H$ is finite, $g_{h m}(i)=d \pm \frac{k}{\sqrt{\text { data }_{i}}}, d$ is a constant greater than 1 and $k$ is a threshold value) ${ }^{[2-3]}$. Determining the position of the peak again: averaging the two points of $g_{h m}(i)$ through 1.Then determine the peak boundary and half-width: the peak boundary is determined by the two extreme ends of the "unit band" lower limit and half-width is determined by t the intercept of $g_{h m}(i)$ at "1"; Finally, determine the combined peak: there is no "in band" product function value between the two peaks of product function.

The peak-searching process of the Gaussian product function method is shown in Figure 1. The peak-searching sensitivity is related to $m$, and the sensitivity of peak-searching increases with $m$. As 
$m$ increases, the peak value of the Gaussian product function moves toward the lower track, resulting in a larger deviation between the experimentally searched peak positions and the actual peak positions, this is not suitable for determining the peak position. In the situation of weak radioactivity, some channel positions may have very small counts or even zero counts. When using the Gaussian product function method to peak-searching, there may be a meaningless situation. Therefore, the data must be smoothed before using Gaussian product function method to peaksearching. Furthermore, $m, k$ should be selected reasonably, it is better to choose $m=2$ and $k=3$ after testing.

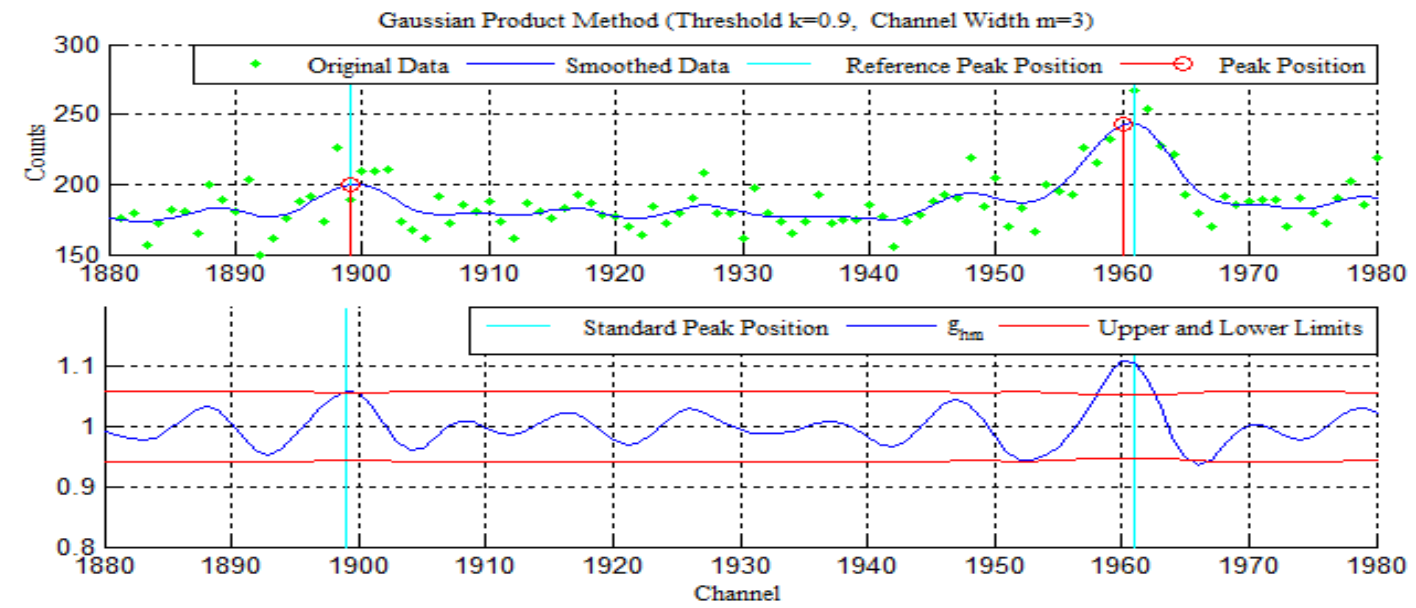

Figure 1. Schematic diagram of peak-searching process of Gaussian product function

\section{The peak-searching of symmetrical zero area Gaussian function method}

\subsection{Symmetric zero area Gaussian function method}

The basic idea is that using the convolution transformation between the zero-area "window" Function and the experimental spectrum data, the "window" function is required to be a symmetric function. The convolutional transformation of a linear substrate will be zero, and only the convolutional transformation of the peak positions is not zero. Therefore, the change in peak shape can be reflected by the symmetric zero-area conversion spectrum of the original spectral data. Its mathematical expression is as follows ${ }^{[2-5]}$ :

$$
B_{i}=\sum_{j=-m}^{m} A_{j} \cdot \operatorname{data}_{i+j}, \sum_{j=j-m}^{m} A_{j}=0, A_{j}=A_{-j}
$$

In the above equation, $B_{i}$ is the transformed spectral data, $d a t a_{i}$ is the original experimental spectral data, $A_{j}$ is the symmetric zero area transform function, and $\mathrm{W}=2 m+1$ is called the window width, i.e. the transform width.

Select the difference between a specific function and a constant $\mathrm{C}$ as a symmetric zero area transformation function:

$$
A_{j}=T_{j}-C, \quad C=\frac{1}{W} \sum_{j=-m}^{m} T_{j}
$$

In the above formula, $T_{j}$ is a specific function, Gauss function $T_{j}=\exp \left[-4 \ln 2(j / H)^{2}\right]$ or Gaussian function second-order derivative $T_{j}=\frac{8 \ln 2}{H^{4}}\left(8 \ln 2 j^{2}-H^{2}\right) \exp \left[-4 \ln 2(j / H)^{2}\right]$ is selected, C is a constant.

In order to make the sensitivity of peak-searching unaffected by the statistics of the experimental spectrum, the standard deviation of the conversion spectrum is generally used as the unit to find the peak. As shown in formula (5): when the ratio of the transformed spectrum to its standard deviation get a positive maximum value, and this value exceeds the given value, the position is considered to be a peak. 


$$
S S_{i}=\frac{B_{i}}{\Delta B_{i}}=\frac{\sum_{j=-m}^{m} A_{j} \cdot \operatorname{data}_{i+j}}{\left(\sum_{j=-m}^{m} A_{j}^{2} \cdot \operatorname{data}_{i+j}\right)^{1 / 2}}>f
$$

The rules of peak-searching: the peak position is determined by the corresponding point of the positive maximum value; $f$ is the peak-searching threshold or sensitivity factor, which is a positive constant; the peak boundary is determined by the adjacent negative peaks on both sides of the positive peak of $S S_{i}$; the two zero-crossing intercepts are half-width $\mathrm{H}$, which can be expressed by the full width at half maximum (FWHM) of the experimental spectrum.

\subsection{The peak-searching process of Symmetric zero area Gaussian function method}

The symmetric zero-area Gaussian function peak-searching process is shown in Figure 2. The specific peak-searching process is described as follows: first, the transform spectrum is calculated with a larger window width $(w)$, the possibility of overlapping peaks is estimated from the distances intercepted by zero-crossing on both sides of the positive region. In general, if the two zero-crossing intercepts exceed FWHM, it is determined that the position have overlapping peaks. then, using a small window width $(w)$ to calculate the conversion spectrum, the number of peaks with a positive conversion spectrum is the number of overlapping peaks, and the positive peak position is the position of each overlapping peak.

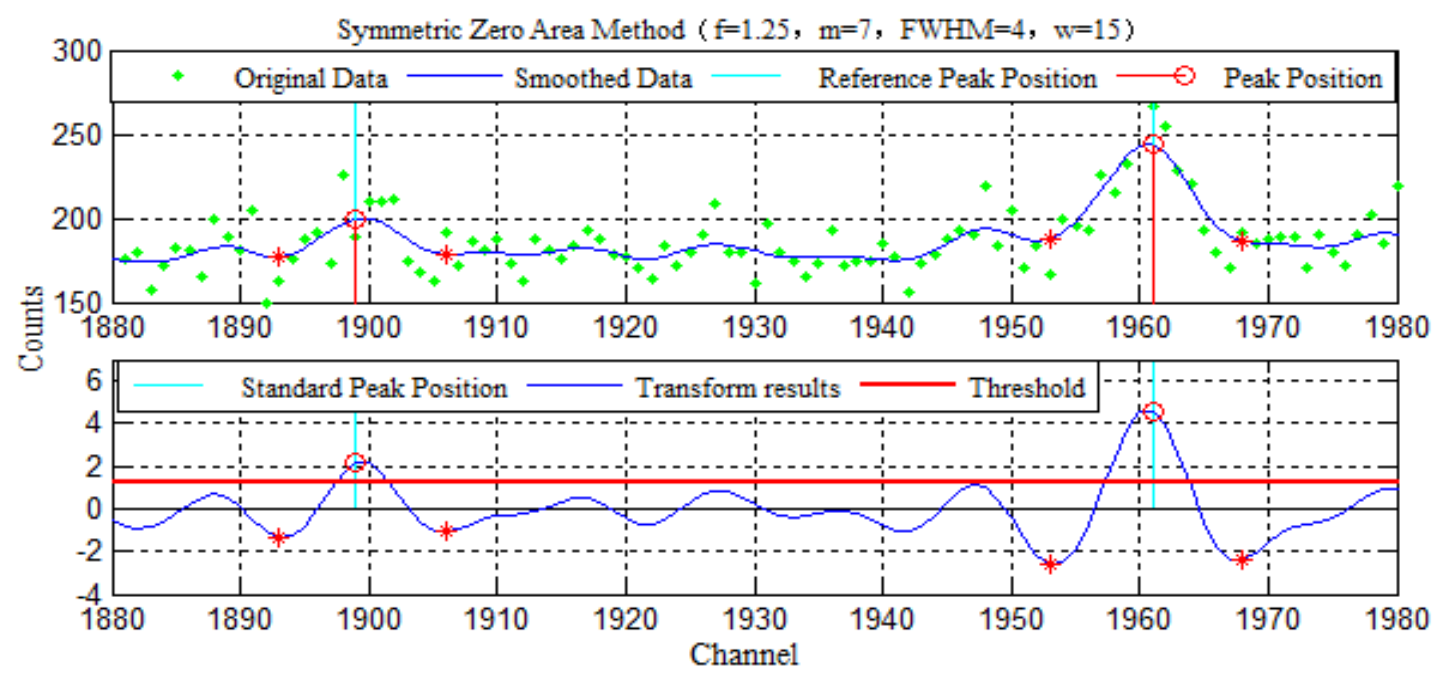

Figure 2. Schematic diagram of the peak searching process of symmetric zero area Gauss function method

The symmetric zero-area Gaussian function is the best transformation function by comparing the aforementioned peak-searching process of the Gaussian product function method. Since the Gaussian product function method has the spectrum data as the denominator, the method should not be used before the spectral line is smoothed. If the researchers uses unsmooth data, the Gaussian product function method is susceptible to statistical fluctuations, resulting in poor peak-searching results ${ }^{[6-7]}$. The symmetric zero area Gauss function method can also be applied to unsmooth data, this method has a small amount of calculation and a fast search speed. However, there are many parameters for peak searching sensitivity. For example, the larger the window width $w$, the stronger the statistical fluctuation suppression ability, but the worse the distinguishing ability of overlapping peaks.

\section{The combined peak-searching for symmetric zero area and Gaussian product function}

\subsection{G-1 test spectrum description}

The G-1 test spectrum contains 9 spectra, of which G-1100 is the reference spectrum, G-1200 is the peak-searching test spectrum, G-1300-G-1305 is the conformance test spectrum and G1400 is deconvolution test spectrum ${ }^{[8-9]}$. These spectra are all derived from the comparison of spectral lines 
by the IAEA 1976. Spectra are obtained by accurate measurement of pure nuclear equipment (counting less than 1 million at the highest point of each spectral line). The $60 \mathrm{~cm}^{3} \mathrm{Ge}$ (Li) detector, the FWHM of the $1332 \mathrm{keV}$ peak is $2.8 \mathrm{keV}$, the peak-to-Compton ratio is $40: 1$, the peak has a very small asymmetry, the spectral line is recorded in $2000 \mathrm{ch}$ with gain $0.5 \mathrm{keV} / \mathrm{ch}$, and the range of the spectral line is approximately $1000 \mathrm{keV}$. The FWHM of the $1332 \mathrm{keV}$ peak is approximately $5.6 \mathrm{ch}$. The task of the test is to find the true peaks in all spectrums without detecting false peaks. Then, the peak area is obtained by deconvolution operation, then using the peak position and peak area to compare with expected values to get the performance standard of the test method.

\subsection{Cases analysis of combined peak-searching using symmetric zero area and Gaussian product function}

The G-1100 spectrum contains 20 peaks considered as individual nuclides, this spectrum is the accumulation of the full spectrum of all 20 virtual peaks on the whole energy segment, each peak contains approximately 65,000 counts and approximately $0.4 \%$ of the measurement uncertainty. G1200 contains 22 peaks, most of which are difficult to detect and measure, it is obtained through the shift and attenuation of the G-1100 test spectrum. G-1200 can be used as a test peak-searching method and test algorithm to calculate the spectral peak area. Experience shows that most algorithms usually detect up to 17 spectral peaks and there are 1-2 false peaks. The peak-searching data of the G-1200G test spectrum is shown in Table 1.

Table 1. The peak-searching data table of the G-1200 test spectrum

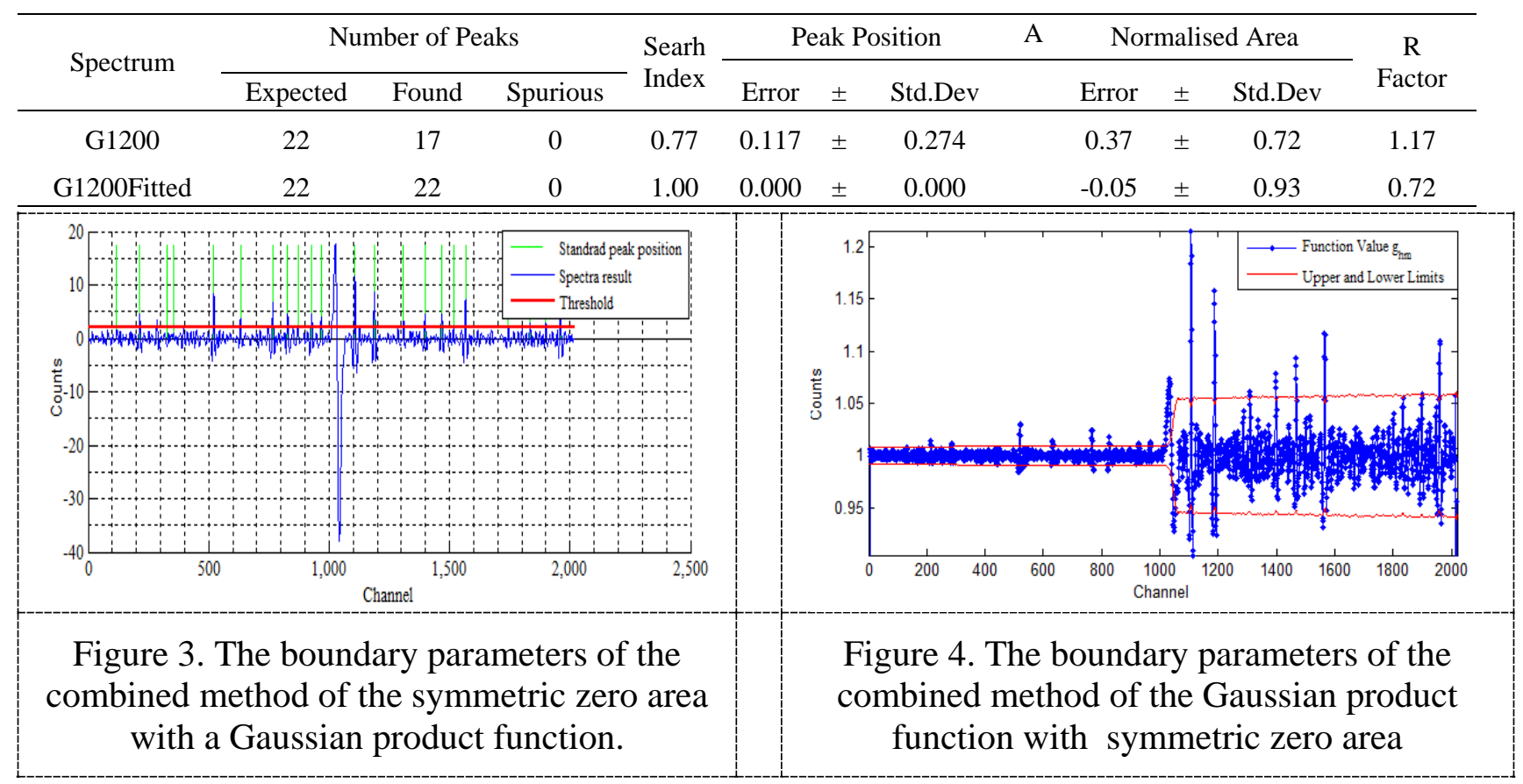

The boundary parameters of the combined method of the symmetric zero area with a Gaussian product function are shown in Figure 3 and Figure 4. The combined method of the symmetric zero area with a Gaussian product function contains boundary parameters such as window width $(w)$, threshold ( $f$ ), full width at half maximum (FWHM), and channel width $(m)$, the inhibitory ability of statistical fluctuations and the resolving ability of overlapping peaks are affected by the window width, the boundary of the combined peak is determined by the FWHM, and it is difficult to adjust sensitivity parameters. The Gaussian product function method is very sensitive to statistical fluctuations, the result of the peak-searching test with the G-1200 spectrum are general, the peaksearching sensitivity is related to the parameter $m$, as the $m$ increases, the peak-searching sensitivity increases. However, the improved combined peak-searching method only needs to determine the parameters such as $w, f$, FWHM, and $m$.

The result of combined peak-searching method of symmetric zero area with a Gaussian product function are shown in Figure 5. The half-width $(H)$ of the Gaussian transform function is 4, that is, 
FWHM=4, the window width $(w)$ is $19 \mathrm{Ch}$, the peak searching threshold $(f)$ is 2.01 , the transition spectrum is more prominent in the "true" peak position when the channel width $(m)$ is 9 , statistical false peaks are suppressed and the peak-searching effect is the best. Moreover, if $w$ is bigger, the resolution ability of overlapping peaks will be worse. Otherwise, if $w$ is narrower, the resolution of overlapping peaks will be stronger. At the same time, combining the Gauss product function method with 7 point smooth spectral lines, the Gaussian product function formula (2) is modified to become the 11 point formula: $g_{h m}(i)=\frac{\text { data }(i-2) \text { data }(i-1) \text { data }(i+1) \operatorname{data}(i+2)}{\text { data }(i-5) \text { data }(i-4) \text { data }(i+4) \text { data }(i+5)}$, which finds 19 true peaks and 1 false peaks. Since the presence of the Compton effect in the spectral line is the cause of most of the algorithm's errors, this is not a practical feature. For evaluation purposes, peaks reported in the 1020 $\mathrm{keV}-1030 \mathrm{keV}$ range are identified as false peaks and ignored. Therefore, the combined peaksearching method of symmetric zero area with a Gaussian product function achieves a better result in determining the peak position in the gamma energy spectrum analysis.

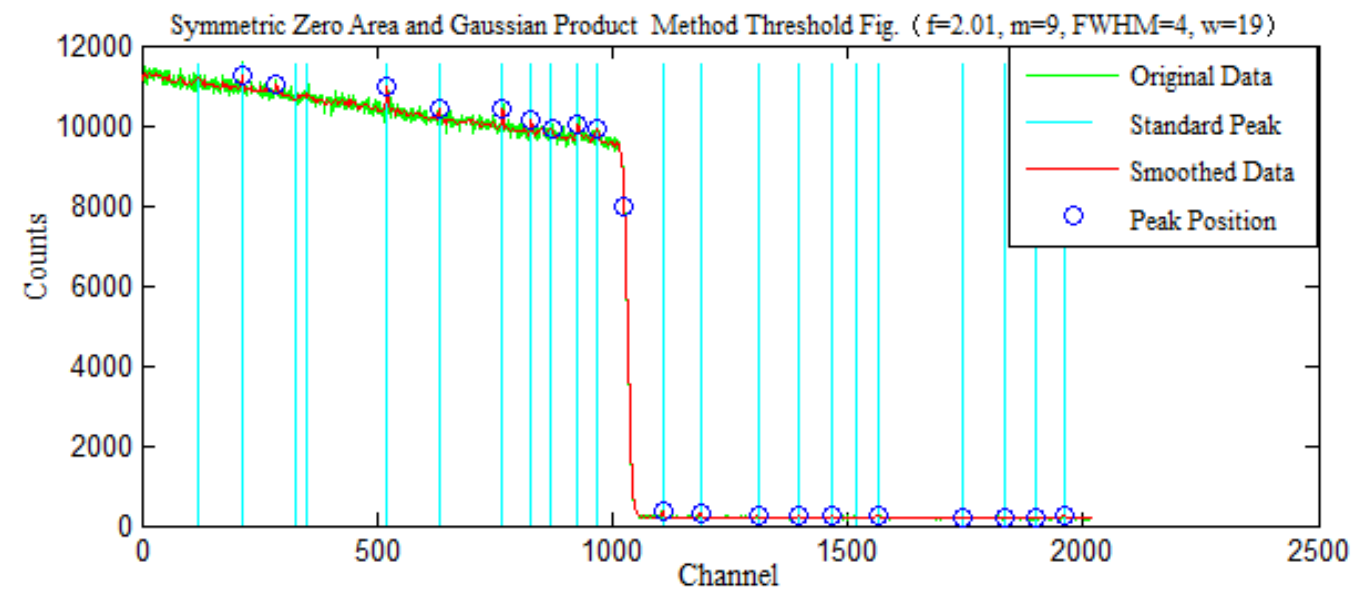

Figure 5. The result of combined peak-searching method of symmetric zero area with a Gaussian product function

\section{Conclusion}

In summary, radionuclides have inherent characteristic peaks, the type of radionuclides can be known by measuring the energy of the characteristic peaks (channel position). Therefore, the accurate determination of the peak positions in gamma spectroscopy are the basis for qualitative analysis. By comparison of the Gaussian product function peak-searching method and the symmetrical zero-area method peak-searching method, the Gaussian product function method is not applicable to the weaker spectral peaks before the spectral data is smoothed. If the spectral data is smoothed before peak searching, the decomposition of overlapping peaks will be affected. However, using the Gaussian function product method, the combined peak boundary is easily determined when the peak area is determined, sensitivity constants such as upper and lower limits are selected to suppress spurious peaks. From the aspects of high background suppression ability, weak peak recognition accuracy and resolving ability of overlapping peaks, the symmetric zero area transformation method has better peak searching effect. However, since the type of peak needs to be determined by comparing the distance between the two peaks and the FWHM, it is necessary to select more characteristic parameters when using the symmetrical zero-area method for peak searching. Regardless of the statistical characteristics of the peaks, the combined peak-searching method of symmetric zero area with a Gaussian product function can be used, this method has a small amount of calculation, fast peak searching speed and obvious results.

\section{Acknowledgments}

This work was supported by the National Natural Science Foundation of China (No.11865002 and No.41862012), the Jiangxi Province Natural Science Foundation of China 
(No.20161BAB201035), the Science and Technology Project on Jiangxi Province Educational Commission of China (No.GJJ160556 and GJJ170642), the Fundamental Science on Radioactive Geology and Exploration Technology Laboratory, East China University of Technology (No.RGET1609), and Fundamental Science on Radioactive Geoscience and Big Data Technology, East China University of Technology (No. JELRGBDT201703).

\section{References}

[1] Gilmore G R. Practical Gamma-Ray Spectrometry (Section 15.5.2 in the Second Edition): Second Edition [J]. 2008(7).

[2] J.F. Pang, Gamma Energy Spectrum Analysis (Shanxi Science and Technology Press, Xi'an, 1993), pp. 350-716

[3] Yang Y Z, Fang F, He J F, et al. A Study of the Peak Boundary Method and Effect Evaluation Based on NaI(TI) Detector for $\gamma$-Spectrum Analysis[J]. Applied Mechanics \& Materials, 2013, 347350:1006-1011.

[4] Gilmore G R. A least squares spectrum fitting method for the measurement of Ge (Li) gammaray peak areas[J]. Journal of Radioanalytical Chemistry, 1979, 48(1-2):91-104.

[5] JianFeng He, Fang Fang, Yao-Zong Yang, et al. Research on Comparison and Evaluation Studies of Several Smoothing Denoising Method Based on $\gamma$-ray Spectrum Detector[J]. Journal of Harbin Institute of Technology, 2013, 20(2):7-11.

[6] He J F, Yang Y Z, Qu J H, et al. An inversion decomposition method for better energy resolution of $\mathrm{NaI}(\mathrm{Tl})$ scintillation detectors based on a Gaussian response matrix[J]. Nuclear Science and Techniques, 2016, 27(3):58-67.

[7] $\mathrm{Xu} \mathrm{H} \mathrm{K,} \mathrm{Fang} \mathrm{F,} \mathrm{Ni} \mathrm{S,} \mathrm{et} \mathrm{al.} \mathrm{Gamma-Ray} \mathrm{Spectrum} \mathrm{Analysis} \mathrm{of} \mathrm{Chang'E-1} \mathrm{for} \mathrm{Lunar}$ Detection[J]. Journal of Engineering for Gas Turbines \& Power, 2011, 133(5):49-54.

[8] Parr R M, Houtermans H, Schaerf K. IAEA intercomparison of methods for processing Ge(Li) gamma-ray spectra[J]. Trans. Am. Nucl. Soc. Suppl.; (United States), 1978, 28:1:49-49.

[9] Zagyvai P, Parr R M, Nagy L G. Additional results for the “G-1" IAEA Intercomparison of Methods for Processing Ge(Li) Gamma-Ray Spectra[J]. Journal of Radioanalytical \& Nuclear Chemistry, 1985, 89(2):589-607. 